\title{
A Unified Framework for Linear Network
}

\section{Coding}

\author{
Min Tan, Raymond W. Yeung, Siu-Ting Ho \\ Department of Information Engineering \\ The Chinese University of Hong Kong \\ New Territories, Hong Kong, China \\ Email: mtan6,whyeung,stho3@ie.cuhk.edu.hk
}

\begin{abstract}
A condition which governs the possibility and impossibility of linear independence among the global encoding kernels of a linear network code is found. Based on this condition, we proposed several alternative definitions of generic network codes, which give interpretations of such codes from different perspectives. We also present a unified framework for specifying and constructing different classes of linear network codes. Finally, using the insights obtained from the unified framework, we show that the proofs of some existing results regarding generic network codes can be greatly simplified.
\end{abstract}

\section{INTRODUCTION}

In Ahlswede et al. [1], the concept of network coding was introduced and the capacity of the single-source multicast network was found. Following [1], Li et al. [2] proved that this singlesource multicast capacity can be achieved by linear network codes (see also Koetter and Medard [3]). Generic network codes were introduced as capacity-achieving codes in the same paper. Jaggi et al. [4] further proved that capacity achieving linear network codes can be constructed in polynomial time. Ho et al. [5] showed that random linear network codes can achieve multicast capacity with high probability provided the field size is large enough. Yeung et al. [6] defined different classes of linear network codes, namely generic network code, linear dispersion, linear broadcast, and linear multicast in decreasing strength, with linear multicast being equivalent to the code constructed in [4]. They also provided a polynomial-time construction algorithm for 
generic network codes. Kwok and Yeung [7] proved a relationship between generic network code and linear dispersion. We refer the reader to Yeung [8] for a detailed discussion of the above.

The original definition of generic network code in [2] is in terms of abstract algebra, making it conceptually difficult to understand. Also, this definition does not facilitate the verification of such codes. These two points will be explained in detail in the later part of this paper. Thus, we are motivated to further investigate this concept with the aim to make it more transparent. As we will see, this leads to alternative definitions of generic network codes that turns out to be useful in different contexts.

The main contributions of this paper are summarized in the following:

1) Fundamental concepts regarding linear independence among global encoding kernels are studied in depth and a condition that governs the possibility and impossibility of linear independence among global encoding kernels is given.

2) Based on the condition in (1), the relationship between generic network codes and graph theory is established and alternative definitions of generic network codes are presented.

3) A unified framework for linear network codes based on the condition in (1) is presented.

4) Some exiting results whose original proofs were complicated can be greatly simplified by using this unified framework.

This paper is organized as follows. In Section 2, the basic concepts of linear network codes are reviewed and some new definitions are introduced for the convenience of discussion. In Section 3, generic network codes are revisited; the disadvantages of the original definition of such codes are discussed; new definitions of generic network codes are introduced and their equivalence to the original definition is proved. We also use the insight developed in here to simplify the proof of some existing results regarding generic network codes. The conclusion of this paper is in Section 4.

\section{PRoblem Formulation}

A communication network is modeled as a finite directed graph $\mathcal{G}=(\mathcal{V}, \mathcal{E})$ where $\mathcal{V}$ is a set of nodes and $\mathcal{E}$ is a set of edges connecting these nodes. A edge in $\mathcal{E}$ will also be referred to as a channel. A node is called a source node if it does not contain any incoming edge; a node is called a sink node if it does not contain any outgoing edge. If the communication network 
does not contain any directed cycle, then it is called an acyclic network. Otherwise, it is called a cyclic network. If the communication network contains only one source node, then it is called a single-source network. If it contains multiple sources, then it is called a multi-source network. The discussion in this paper is restricted to single-source acyclic networks. The unique source node is denoted by $s$ and the set of all sink nodes is denoted by $T$. At the source node $s$, information to be transmitted across the network is generated. To facilitate our discussion, we assume that multiple edges are allowed between nodes and each edge has unit capacity, which means that one symbol taken from a certain finite field $G F(q)$ can be transmitted over each edge. This assumption is general because we can always quantize the capacity to arbitrary degree of accuracy and represent it by multiple edges. We denote by $\operatorname{In}(v)$ the set of incoming edges of node $v$ and $\operatorname{Out}(v)$ the set of outgoing edges of node $v$. We denote by Tail $(e)=t$ if edge $e$ is an outgoing edge of node $t$ and by $\operatorname{Head}(e)=t$ if edge $e$ is an incoming edge of node $t$.

Let the information to be transmitted from the source node be represented by a row vector $x$ which consists of $\omega$ symbols in $G F(q)$. Following [6], we install a set of $\omega$ incoming imaginary edges at $s$ and associate each of them with a distinct vector in an $\omega$-dimensional standard basis. These vectors are refereed to as the global encoding kernels of the imaginary edges.

The set of all local encoding kernels $k_{d, e} \in G F(q)$, where $d \in \operatorname{In}(v)$ and $e \in O u t(v)$ for some $v \in \mathcal{V}$, specifies a linear network code. For each edge $e$ other than an imaginary edge, we iteratively define its global encoding kernel by

$$
f_{e}=\sum_{d \in \operatorname{In}(t)} k_{d, e} f_{d}
$$

where $t=\operatorname{Tail}(e)$. In other words, at each intermediate node, the incoming global kernels are linearly combined to produce the outgoing global encoding kernels. The received information symbol at each edge $e$ can be calculated as $x \cdot f_{e}$.

For a collection of nodes $T$, we define

$$
V_{T}=\left\langle f_{e}: \operatorname{Head}(e) \in T\right\rangle
$$

For a set of edges $E$, we denote their corresponding global encoding kernels by

$$
K(E)=\{f(e): e \in E\}
$$


A sequence of edges $e_{1}, e_{2}, \ldots, e_{n}$, where $e_{1}$ may be an imaginary channel, form a path if $\operatorname{Head}\left(e_{i}\right)=\operatorname{Tail}\left(e_{i+1}\right)$ for $1 \leq i \leq n-1$. Two paths are edge-disjoint if they do not have any edge in common.

A set of edges is an independent set ${ }^{1}$ if each edge is on a path originating from an imaginary channel (i.e., the first edge of the path is an imaginary channel) and these paths are edge-disjoint. We call this set of paths an associated flow for this independent set. An independent set may have more than one associated flows. Note that an independent set concerns only the position of edges in the graph but not the global encoding kernels that may be assigned to them.

For a linear network code defined on the acyclic network, if the corresponding global encoding kernels of an independent set are linearly independent, then we say that this independent set is regular. For any collection of edges $a, b, e_{1}, e_{2}, \ldots, e_{i}$ where $i \geq 0$, if $\operatorname{Head}(a)=\operatorname{Tail}(b)$ and $\alpha=\left\{a, e_{1}, e_{2}, \ldots, e_{i}\right\}$ and $\beta=\left\{b, e_{1}, e_{2}, \ldots, e_{i}\right\}$ are independent sets, then independent set $\alpha$ is said to support independent set $\beta$ and we denote it by $\alpha \rightarrow \beta$. The above concepts are illustrated in the following example.

Example 1: Figure 1 shows a single-source linear network code. We observe that edge $e_{13}$ can be traced back to the imaginary channel via the reversed path $P_{1}=e_{13}, e_{7}, e_{2}, e_{s 2}$ and edge $e_{14}$ can be traced back to the imaginary channel via the reversed path $P_{2}=e_{14}, e_{10}, e_{3}, e_{s 1}$. These two paths are edge-disjoint. Thus, $\left\{e_{13}, e_{14}\right\}$ forms an independent set and $\left\{P_{1}, P_{2}\right\}$ is an associated flow for this independent set. Here $f_{e_{14}}$ and $f_{e_{13}}$ are linearly independent, and so $\left\{e_{13}, e_{14}\right\}$ is a regular independent set.

Now let us look at edges $e_{3}$ and $e_{10}$. Edge $e_{3}$ is the only upstream edge of edge $e_{10}$ and any reverse path from edge $e_{10}$ to the imaginary channel must also pass through $e_{3}$. Thus edge $e_{3}$ and edge $e_{10}$ do not form an independent set. We note that edge $e_{13}$ can also be traced back to the imaginary channel by reverse path $P_{3}=e_{13}, e_{5}, e_{1}, e_{s 2}$, and $P_{3}$ and $P_{2}$ are edge-disjoint. Thus, $\left\{P_{2}, P_{3}\right\}$ forms another associated flow for the independent set $\left\{e_{13}, e_{14}\right\}$. It is not difficult to verify that $e_{8}$ and $e_{10}$ also form an independent set with a unique associated flow.

Finally, the global encoding kernels of an independent set are not necessarily linearly independent. For example, $\left\{e_{12}, e_{13}\right\}$ is an independent set, but their global encoding kernels are linearly dependent. We observe that both $\left\{e_{8}, e_{10}\right\}$ and $\left\{e_{8}, e_{3}\right\}$ are independent sets and

\footnotetext{
${ }^{1}$ This name is justified in a separate paper by Sun et al. [9] which explicitly defines the underlying matroid structure.
} 


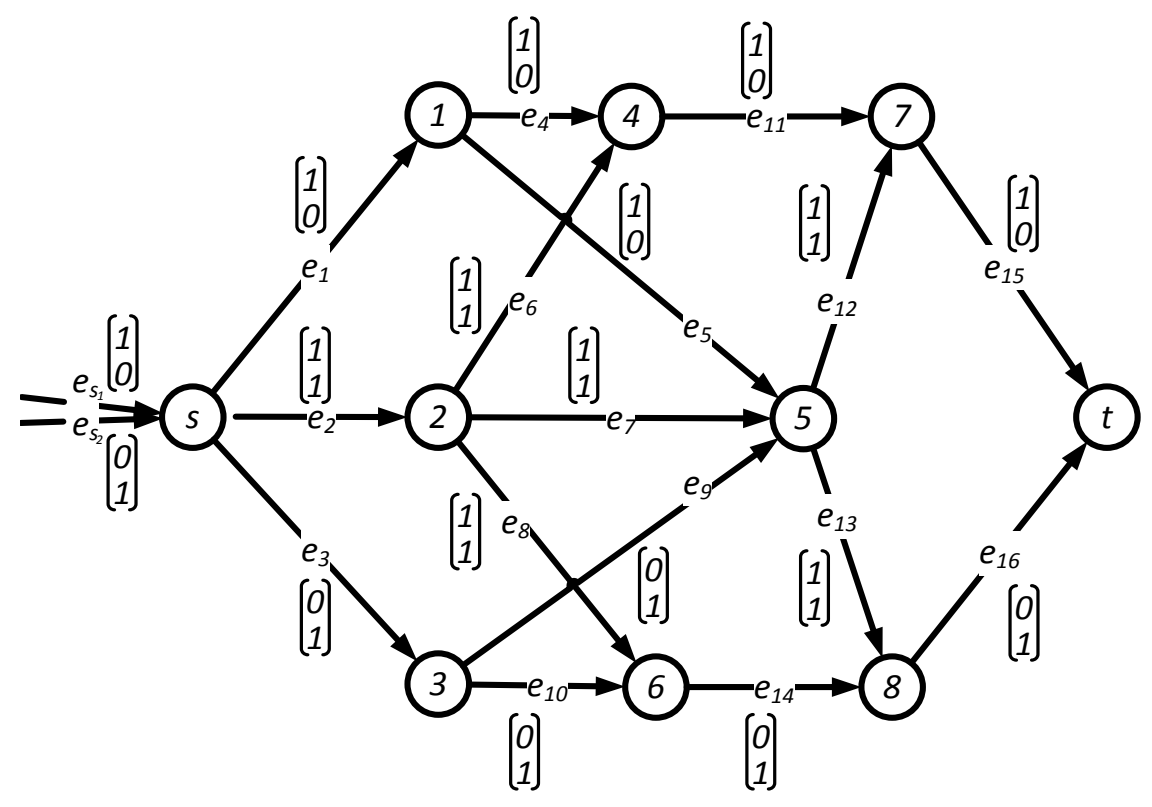

Fig. 1. Independent set and associated flow

$\operatorname{Head}\left(e_{3}\right)=\operatorname{Tail}\left(e_{10}\right)$. Thus, $\left\{e_{8}, e_{3}\right\}$ supports $\left\{e_{8}, e_{10}\right\}$, i.e. $\left\{e_{8}, e_{3}\right\} \rightarrow\left\{e_{8}, e_{10}\right\}$.

\section{UNIFIED FRAMEWORK}

\section{A. Generic Network Codes Revisited}

Generic network codes were first introduced in Li et al. [2] as a way to achieve the multicast capacity in a single-source network. A construction algorithm of generic network code is also proposed in that paper. The original definition of generic network codes is reproduced below for convenience.

Definition 1: An $\omega$-dimensional linear network code on a single-source acyclic communication network is said to be generic if the following condition holds for any collection of edges $e_{1}, e_{2}, \ldots, e_{m}$ for $1 \leq m \leq \omega: V_{\text {Tail }\left(e_{k}\right)} \not \subset\left\langle f_{e_{j}}: j \neq k\right\rangle$ for $1 \leq k \leq m$ if and only if the vectors $f_{e_{1}}, f_{e_{2}}, \ldots, f_{e_{m}}$ are linearly independent.

This definition has several disadvantages. First, it is conceptually difficult to be understood. It was mentioned in [6] that the motivation for generic network codes is to define a linear 
network code such that every collection of global encoding kernels that can possibly be linearly independent must be linearly independent. However, it is not clear from [6] what it means by a collection of global encoding kernels being possibly linearly independent. One goal of this paper is to establish the connection between linear independence among global encoding kernels and generic network codes. As we will see later, this connection allows a more concrete interpretation of generic network codes.

Second, the original definition of generic network code does not facilitate the verification of a generic network code. As we will see, the alternative definitions we will present enables such a verification to be done more efficiently and intuitively.

In this paper, we seek simple characterization for a set of global encoding kernels to be possibly linearly independent. The lemma below gives the necessary condition for a set of global encoding kernels to be linearly independent.

Lemma 1: If the global encoding kernels of a collection of edges $\left\{e_{1}, e_{2}, \ldots, e_{m}\right\}$, where $1 \leq$ $m \leq \omega$, are linearly independent, then each edge is on some path originating from an imaginary channel and these paths are edge-disjoint, namely these edges form an independent set.

Proof: Consider a collection of edges $\left\{e_{1}, e_{2}, \ldots, e_{m}\right\}, 1 \leq m \leq \omega$, whose global encoding kernels are linearly independent. We connect $\operatorname{Tail}\left(e_{i}\right)$ to a new node $t$ by a new edge $e_{i}^{\prime}$ for $1 \leq i \leq m$, respectively and let $f_{e_{i}^{\prime}}=f_{e_{i}}$ for $1 \leq i \leq m$. Consider any cut $U$ between the source $s$ and node $t$ and let $E_{U}$ be the set of edges across the cut $U$. We denote by $\operatorname{Mincut}(\mathrm{s}, \mathrm{t})$ the min-cut between $s$ and $t$ and by Maxflow(s,t) the max-flow between $s$ and $t$. Then $V_{t}$ is a linear transformation of $\operatorname{span}\left(K\left(E_{U}\right)\right)$, where

$$
\operatorname{dim}\left(V_{t}\right) \leq \operatorname{dim}\left(\operatorname{span}\left(K\left(E_{U}\right)\right)\right) \leq\left|E_{U}\right|
$$

It follows that

$$
\operatorname{dim}\left(V_{t}\right) \leq \min _{U}\left|E_{U}\right|=\operatorname{Mincut}(\mathrm{s}, \mathrm{t})
$$

In particular, for the cut $U^{*}$ between $s$ and $t$ such that $E_{U^{*}}=\left\{e_{i}^{\prime}: 1 \leq i \leq m\right\}$, we have

$$
m=\operatorname{dim}\left(V_{t}\right) \leq \operatorname{Mincut}(\mathrm{s}, \mathrm{t}) \leq\left|E_{U^{*}}\right|=m
$$

Thus, Maxflow $(\mathrm{s}, \mathrm{t})=\operatorname{Mincut}(\mathrm{s}, \mathrm{t})=m$ by the Max-flow Min-cut theorem and $t$ can always be traced back to imaginary channels by a set of edge-disjoint paths. Changing the last edges in 
these edge-disjoint paths from $e_{i}^{\prime}$ to $e_{i}$ for $1 \leq i \leq m$, we obtain the desired set of edge-disjoint paths. We can always do so because $\operatorname{Tail}\left(e_{i}^{\prime}\right)=\operatorname{Tail}\left(e_{i}\right)$.

The above lemma says that a collection of global encoding kernels can possibly be linearly independent only if their corresponding edges form an independent set. Thus the best linear network code we can hope for in terms of linear independence is the one in which a collection of global encoding kernels are linearly independent whenever the corresponding edges form an independent set. In designing a linear network code, if the global encoding kernels are required to be independent on only one independent set, it can be achieved by routing alone. This is illustrated by the example in Figure 2. For instance, the global encoding kernels of the incoming edges of node 3 and node 4 can be made linearly independent simply by routing the 2 source symbols to node 3 and node 4, respectively.

If the global encoding kernels are required to be linearly independent on multiple independent sets, since these independent sets may couple with each other through their common edges, routing in general will fail to achieve the desired linear independence. This is illustrated in Figure 3 . Here, independent set 1 consists of three edges, and independent set 2 consists of two edges. If these two independent sets are regular, then $f_{e_{11}} \neq f_{e_{12}}$, because $f_{e_{12}} \neq f_{e_{16}}$ and $f_{e_{11}}=f_{e_{16}}$. If we do not encode at node $R_{5}$, then $f_{e_{12}}=f_{e_{8}}$ implies that $f_{e_{7}}=f_{e_{11}}$ which in turn implies that $f_{e_{10}}=f_{e_{15}}$. Thus independent set 1 fails to be regular. Because of the coupling between independent set 1 and independent set 2, routing fails to achieves the desired linear independence.

The situation may change if coding is allowed at the intermediate nodes. An interesting question to ask is whether we can always construct a linear network code in which the global encoding kernels of every independent set are linearly independent. The following lemma provides a positive answer to this question.

Lemma 2: For any collection of independent sets $\mathcal{I}$, there always exists a linear network code such that any independent set in $\mathcal{I}$ is regular provided $q \geq|\mathcal{I}|$, where $q$ is the size of the base field.

Proof: We specify the global encoding coding kernels iteratively as in the Jaggi-Sanders algorithm [4]. By definition, each independent set in $\mathcal{I}$ has an associated flow. Initially, only the global encoding kernels of the imaginary channels, namely the standard basis, are specified. In 


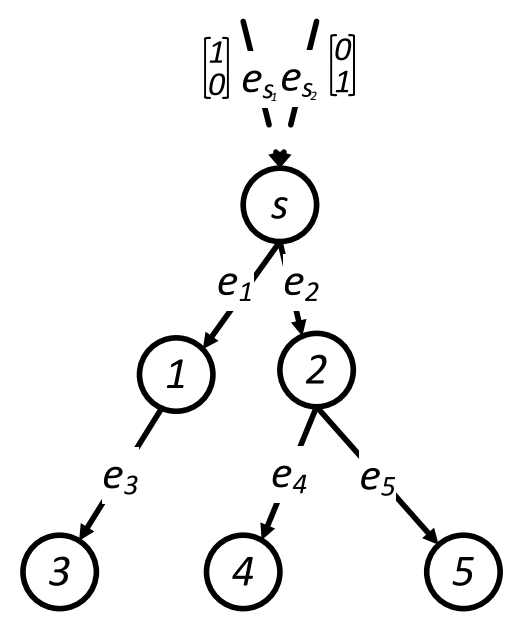

Fig. 2. For single independent set, linear independence can be achieved by routing alone.

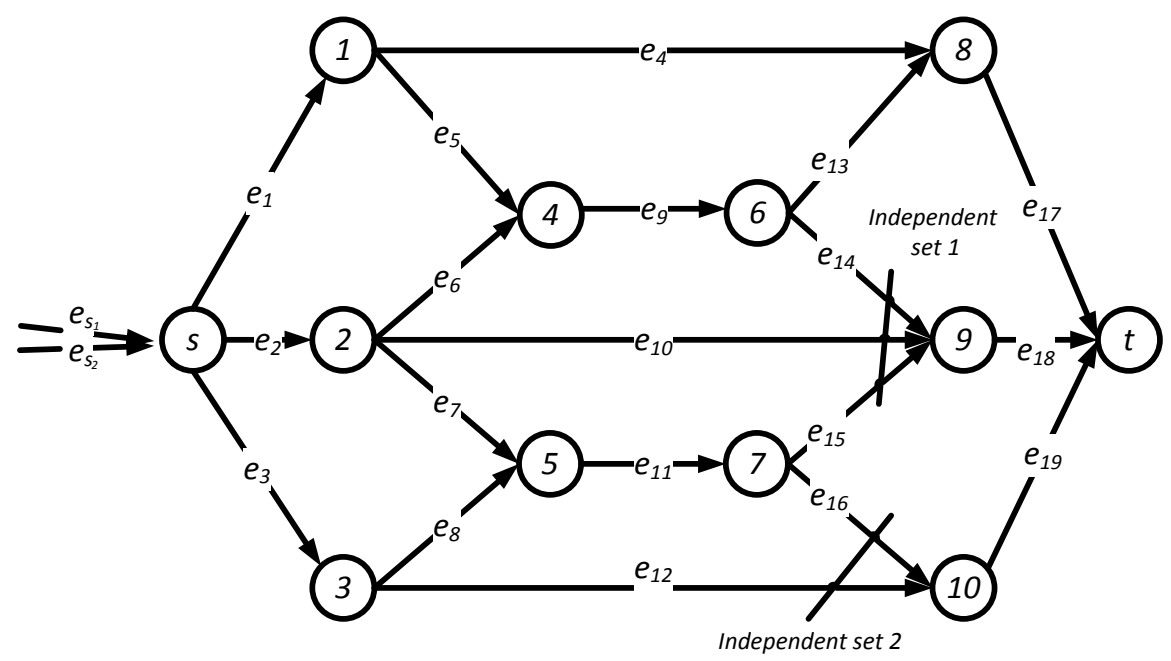

Fig. 3. Routing in general fails to achieve the desired independence for multiple independent sets. 
our algorithm, the global encoding kernels are specified in an upstream-to-downstream manner. For each associated flow, the last processed edges on its paths form a frontier set. Note that a frontier set is an independent set.

In our construction, we are to maintain each frontier set as a regular independent set. At the beginning, the frontier set of each flow associated with each independent set in $\mathcal{I}$ is a subset of all the imaginary channels. Therefore, each frontier set is a regular independent set to start with. Assume that the regularity of all the frontier sets are maintained at the current step. Let $e$ be the next edge to be processed. Let $n$ be the number of new frontier sets induced by edge $e$ and denote these new frontier sets by $\beta_{i}, 1 \leq i \leq n$. Suppose $\alpha_{i} \rightarrow \beta_{i}$ for $1 \leq i \leq n$, where $\alpha_{i}, 1 \leq i \leq n$ are the frontier sets in the current step. Denote by $e_{i}=\alpha_{i} \backslash \beta_{i}$ the only edge that belongs to $\alpha_{i}$ but not $\beta_{i}$ and by $t$ the tail of edge $e$. Since the global encoding kernel of $e_{i}$ and the global encoding kernels of $\alpha_{i} \backslash e_{i}$ are linearly independent for $1 \leq i \leq n$ by the induction assumption and $f_{e_{i}} \in V_{t}$ for $1 \leq i \leq n, V_{t} \backslash \operatorname{span}\left(K\left(\alpha_{i} \backslash e_{i}\right)\right)$ is nonempty. This implies that $\operatorname{dim}\left(V_{t} \cap \operatorname{span}\left(K\left(\alpha_{i} \backslash e_{i}\right)\right)\right) \leq \operatorname{dim}\left(V_{t}\right)-1$ for $1 \leq i \leq n$. If the base field size $q>|\mathcal{I}|>n$, then we have

$$
\begin{aligned}
\left|V_{t} \backslash \cup_{1 \leq i \leq n} \operatorname{span}\left(K\left(\alpha_{i} \backslash e_{i}\right)\right)\right| & =\left|V_{t}\right|-\left|V_{t} \cap\left[\cup_{1 \leq i \leq n} \operatorname{span}\left(K\left(\alpha_{i} \backslash e_{i}\right)\right)\right]\right| \\
& \geq\left|V_{t}\right|-\sum_{1 \leq i \leq n}\left|V_{t} \cap \operatorname{span}\left(K\left(\alpha_{i} \backslash e_{i}\right)\right)\right|+1 \\
& \geq q^{\operatorname{dim}\left(V_{t}\right)}-n \times q^{\operatorname{dim}\left(V_{t}\right)-1}+1 \\
& >q^{\operatorname{dim}\left(V_{t}\right)}-|\mathcal{I}| \times q^{\operatorname{dim}\left(V_{t}\right)-1}+1 \\
& >0 .
\end{aligned}
$$

In the above, the first $\leq$ follows from an application of the union bound and the observation that every subspace contains the origin. Thus, by setting the base field size $q \geq|\mathcal{I}|$, we can always choose the global encoding kernel of $e$ to be a vector in $V_{t} \backslash \cup_{1 \leq i \leq n} \operatorname{span}\left(K\left(\alpha_{i} \backslash e_{i}\right)\right)$ and the regularity of the new frontier sets can be always maintained. Hence, all the independent sets in $\mathcal{I}$ are regular upon the termination of the algorithm.

Lemma 1 and Lemma 2 together implies that there exists a linear network code such that the global encoding kernels of a set of edges are linearly independent if and only if these edges form an independent set. In other words, the independent set governs the possibility and impossibility 
of linear independence among global encoding kernels. The best linear code in terms of linear independence is the one with every independent set being regular. This coincides with the original motivation of generic network code as explained in [6]. In the following, we prove that a linear network code with every independent set being regular is actually a generic network code. We also prove that a generic network code must have every independent set regular. This gives an equivalent definition of generic network codes.

The original definition of generic network codes has an algebraic interpretation, while the equivalent definition gives a graph-theoretic interpretation which provides more intuition. Another equivalent definition that we will prove in the next theorem gives a simpler way to verify whether a linear network code is generic or not. We only consider the case when $\mid$ Out $(s) \mid \geq$ $\omega$, otherwise the problem is degenerate because no node in the network can receive all the information generated at the source node.

Theorem 1: The following five conditions are equivalent for linear network codes with $|O u t(s)| \geq$ $\omega$.

1) For any collection of global encoding kernels $f_{e_{1}}, f_{e_{2}} \ldots f_{e_{m}}$, if $V_{t_{i}} \not \subset\left\langle f_{e_{k}}: k \neq i\right\rangle$ for $1 \leq i \leq m$ where $t_{i}=\operatorname{Tail}\left(e_{i}\right)$ for $1 \leq i \leq m$, then $f_{e_{1}}, f_{e_{2}} \ldots f_{e_{m}}$ are linearly independent.

2) For any collection of global encoding kernels $f_{e_{1}}, f_{e_{2}} \ldots f_{e_{m}}$, if $V_{t_{m}} \not \subset\left\langle f_{e_{1}}, f_{e_{2}} \ldots f_{e_{m-1}}\right\rangle$ and there exists no directed path from $t_{m}$ to $t_{j}$ for $1 \leq j \leq m-1$, where $t_{i}=\operatorname{Tail}\left(e_{i}\right)$ for $1 \leq i \leq m$, then $f_{e_{m}} \notin\left\langle f_{e_{1}}, f_{e_{2}} \ldots f_{e_{m-1}}\right\rangle$.

3) For any collection of global encoding kernels $f_{e_{1}}, f_{e_{2}} \ldots f_{e_{m}}$, if $f_{e_{1}}, f_{e_{2}} \ldots f_{e_{m-1}}$ are linearly independent, $V_{t_{m}} \not \subset\left\langle f_{e_{1}}, f_{e_{2}} \ldots f_{e_{m-1}}\right\rangle$, and there exists no directed path from $t_{m}$ to $t_{j}$ for $1 \leq j \leq m-1$, where $t_{i}=\operatorname{Tail}\left(e_{i}\right)$ for $1 \leq i \leq m$, then $f_{e_{m}} \notin\left\langle f_{e_{1}}, f_{e_{2}} \ldots f_{e_{m-1}}\right\rangle$.

4) For any independent set $\beta$, the global encoding kernels $K(\beta)$ are linearly independent.

5) For any independent set $\alpha$ with $\omega$ edges, the global encoding kernels $K(\alpha)$ are linearly independent.

Remark: Condition 1 is the original definition of generic network codes [6]. Roughly speaking, Condition 2 means that "new" information must be carried by an edge whenever possible. Conditions 4 and 5 give a graph-theoretical interpretation of a generic network code. They say that if a set of edges can be traced back to the imaginary channels via a set of edge-disjoint paths, then their corresponding global encoding kernels must be linearly independent. Though 
these five conditions are equivalent, one condition may be more convenient to use than others in different contexts. For example, Condition 4 provides better intuition. Condition 2 is more useful in constructing such a linear network code. Compared with Condition 4, Condition 5 gives a simpler way for us to verify whether a linear network code is generic or not, for we only need to consider independent sets of size $\omega$.

Proof: We will prove that 5) $\Rightarrow 4) \Rightarrow 3) \Rightarrow 2) \Rightarrow 1) \Rightarrow 5$ ).

$5) \Rightarrow 4$ ): For any independent set $\beta$, we can always enlarge it to an independent set $\alpha$ with $\omega$ edges by including some edges originating from the source node because $\mid$ Out $(s) \mid \geq \omega$. If 5) holds, then the global encoding kernels $K(\alpha)$ are linearly independent. It follows that the global encoding kernels $K(\beta)$ are also linearly independent because $\beta$ is a subset of $\alpha$. Thus 5) implies 4).

$4) \Rightarrow 3$ ): Let $e_{1}, e_{2}, \ldots, e_{m}$ be a set of edges such that $f_{e_{1}}, f_{e_{2}}, \ldots, f_{e_{m-1}}$ are linearly independent, $V_{t_{m}} \not \subset\left\langle f_{e_{j}}: j \neq m\right\rangle$, and there is no directed path from $t_{m}$ to $t_{i}$ for $1 \leq i \leq m-1$, where $t_{i}=$ $\operatorname{Tail}\left(e_{i}\right)$ for $1 \leq i \leq m$. We can always find an edge $e_{m}^{\prime} \in \operatorname{In}\left(t_{m}\right)$ such that $f_{e_{1}}, f_{e_{2}}, \ldots, f_{e_{m-1}}, f_{e_{m}^{\prime}}$ are linearly independent, because $V_{t_{m}} \not \subset\left\langle f_{e_{i}}: i \neq m\right\rangle$. Thus $e_{1}, e_{2}, \ldots, e_{m}^{\prime}$ can be traced back to the imaginary channels via some edge-disjoint paths $P_{1}, P_{2}, \ldots, P_{m}^{\prime}$ respectively by Lemma 1. Because there is no directed path from $t_{m}$ to $t_{i}$ for $1 \leq i \leq m-1$ and $e_{1}, e_{2}, \ldots, e_{m}$ are distinct, $P_{1}, P_{2}, \ldots, P_{m}$, where $P_{m}$ is the path obtained by appending $e_{m}$ to $P_{m}^{\prime}$, must also be edge-disjoint paths. Therefore, $e_{1}, e_{2}, \ldots, e_{m}$ form an independent set. Then $f_{e_{1}}, f_{e_{2}}, \ldots, f_{e_{m}}$ are linearly independent if 4) holds. Thus 4) $\Rightarrow 3$ ).

$3) \Rightarrow 2$ ): Suppose a linear network code satisfies 3). Consider any collection of channels $\xi=\left\{e_{1}, e_{2}, \ldots, e_{m-1}\right\}$ and any channel $e_{m} \notin \xi$ such that $V_{t_{m}} \not \subset\left\langle f_{e_{1}}, f_{e_{2}}, \ldots, f_{e_{m-1}}\right\rangle$, where $f_{e}, e \in \xi$ are not necessarily linearly independent. Then we can always find a subset $\xi^{\prime}$ of $\xi$ such that $V_{\xi}=V_{\xi^{\prime}}$ and $f_{e}, e \in \xi^{\prime}$ are linearly independent. Since the linear network code satisfies 3), we have

$$
f_{e_{m}} \notin V_{\xi^{\prime}}=V_{\xi}
$$

so this linear network code also satisfies 2).

$2) \Rightarrow 1$ ): We prove this by induction on $m$, the number of edges.

a) Let us consider the case $m=2$. Assume 2) holds and consider any collection of global encoding kernels $\left\{f_{e_{1}}, f_{e_{2}}\right\}$ which satisfy 2). Suppose 2) does not imply 1). Then there must 
exist a directed path from $t_{1}$ to $t_{2}$. Otherwise, $f_{e_{1}}$ and $f_{e_{2}}$ would be linearly independent if 2) holds. Similarly, there must exist a directed path from $t_{2}$ to $t_{1}$. But this contradicts the fact that the network is acyclic. Thus our assumption is false, and so 2) implies 1) for $m=2$.

b) Assume 2) $\Rightarrow 1$ ) for $m \leq k$ for some $k \geq 2$. We need to show that 2) $\Rightarrow 1$ ) for $m=k+1$. Consider global encoding kernels $f_{e_{1}}, f_{e_{2}} \ldots f_{e_{k+1}}$ such that $V_{t_{i}} \not \subset\left\langle f_{e_{k}}: k \neq i\right\rangle$ for $1 \leq i \leq k+1$. Assume 2) holds. Denote by $\bar{j}$ the set $\{i: 1 \leq i \leq k+1$ and $i \neq j\}$ for any $1 \leq j \leq k+1$. We observe that $V_{t_{l}} \not \subset\left\langle f_{i}: i \neq l\right\rangle$ and $\left\langle f_{i}: i \in \bar{j}\right.$ and $\left.i \neq l\right\rangle \subseteq\left\langle f_{i}: i \neq l\right\rangle$ for $1 \leq j \leq k+1$ and $l \in \bar{j}$ implies $V_{t_{l}} \not \subset\left\langle f_{i}: i \in \bar{j}\right.$ and $\left.i \neq l\right\rangle$ for $1 \leq j \leq k+1$ and $l \in \bar{j}$. By the induction hypothesis that 2) implies 1), global encoding kernels $\left\{f_{e_{i}}: i \in \bar{j}\right\}$ are linearly independent for $1 \leq j \leq k+1$. If 2) does not imply 1) for $m=k+1$, then, for $\forall 1 \leq i \leq k+1$, there must exist a directed path from $t_{i}$ to some $t_{j}$ where $1 \leq j \leq k+1$ and $i \neq j$. Otherwise, by 2), $\left\{f_{e_{i}}: 1 \leq i \leq k+1\right\}$ would be linearly independent, a contradiction to that 1 ) does not hold for $m=k+1$. Since $k+1$ is a finite number, such directed path would produce a cycle which is a contradiction to the assumption that the network is acyclic. Thus, 2) implies 1) for $m=k+1$.

1) $\Rightarrow 5)$ : Let $\alpha=\left\{e_{1}, e_{2}, \ldots, e_{\omega}\right\}$ be a size $\omega$ independent set. Then there exist $\omega$ edge-disjoint paths $P_{1}, P_{2}, \ldots, P_{\omega}$ from source node $s$ to the channels in $\alpha$, where the last channel on path $P_{i}$ is $e_{i}$. Denote the length of $P_{i}$ by $l_{i}$ and let

$$
L=\sum_{i=1}^{\omega} l_{i}
$$

be the total length of all the paths. We will prove the assertion by induction on $L$. For $L=\omega$, it is easy to check that 1) implies 5), because $\operatorname{Tail}\left(e_{i}\right)=s$ for $1 \leq i \leq \omega$ and $\operatorname{dim}\left(V_{s}\right)=\omega$. Suppose $K(\alpha)$ is linearly independent for any $\alpha$ with $\omega \leq L \leq k$, where $k \geq \omega$. We will prove that $K(\alpha)$ is linearly independent for any $\alpha$ with $L=k+1$. Let $A=\left\{i: l_{i}>1\right\}$ and $\alpha_{i}=\left\{e_{1}, e_{2}, \ldots, e_{i-1}, e_{i}^{\prime}, e_{i+1}, \ldots, e_{\omega}\right\}$ for $i \in A$, where $e_{i}^{\prime} \in P_{i}$ and $\operatorname{Head}\left(e_{i}^{\prime}\right)=\operatorname{Tail}\left(e_{i}\right)$. Then, for $\alpha_{i}$ where $i \in A$, the global encoding kernels $K\left(\alpha_{i}\right)$ are linearly independent by the induction hypothesis, which implies $V_{t_{i}} \not \subset\left\langle f_{e_{k}}: k \neq i\right\rangle$. This implies that for such an $i \in A$, we have $V_{i} \not \subset\left\langle f_{e_{k}}: k \neq i\right\rangle$. On the other hand, for any $1 \leq i \leq \omega$ and $i \notin A$, we have $V_{t_{i}}=V_{s} \not \subset\left\langle f_{e_{k}}: k \neq i\right\rangle$. It follows that $V_{t_{i}} \not \subset\left\langle f_{e_{k}}: k \neq i\right\rangle$ for all $1 \leq i \leq \omega$. If 1) holds, then the global encoding kernels $K(\alpha)$ are linearly independent and we have finished the induction. Thus 1) implies 5). 


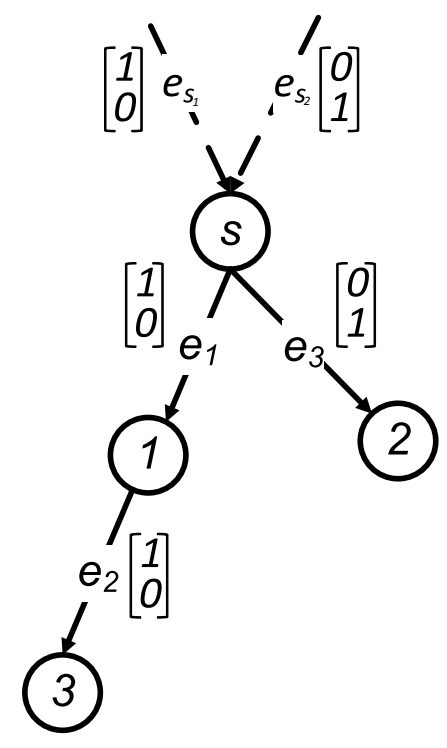

Fig. 4. Graph with directed path

We note that 1$) \Rightarrow 5$ ) was previously proved in the full version of [10]. The condition that there exists no directed path from $t_{m}$ to $t_{j}$ for $1 \leq j \leq m-1$ in 3 ) is essential. Otherwise, the equivalence of the various conditions in the theorem may fail to hold. This is illustrated in Figure 4. We can verify that this linear network code is a generic network code. It is not difficult to verify that 4$)$ holds. We observe that $V_{\text {Tail }\left(e_{1}\right)} \not \subset\left\langle f_{e_{2}}\right\rangle$, but the global encoding kernel $f_{e_{1}} \in\left\langle f_{e_{2}}\right\rangle$. Thus, 3) does not hold if we do not impose the constraint that there is no direct path from $e_{1}$ to $e_{2}$.

It is also interesting to note that from 5), we can construct a generic network code by considering only the independent sets with $\omega$ edges. In this case, the required field size according to Lemma 2 is $\left(\begin{array}{c}|\mathcal{E}| \\ \omega\end{array}\right)$ where $|\mathcal{E}|$ is the number of edges in the network.

\section{B. Unified Framework}

According to the definition in [6], a linear dispersion, a linear broadcast, or a linear multicast is characterized by the dimension of the span of the incoming global encoding kernels associated with certain collections of nodes. For example, for a linear multicast, any non-source node $t$ with $\operatorname{maxflow}(t) \geq \omega$ has $\operatorname{dim}\left(V_{t}\right)=\omega$. For a linear broadcast, any collection of non-source 


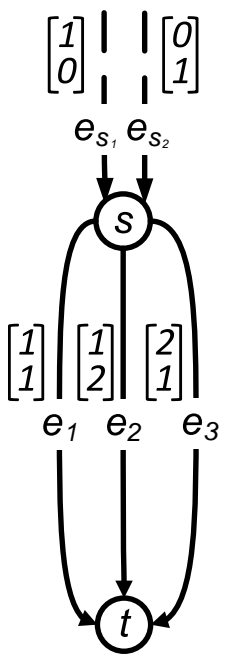

(a)

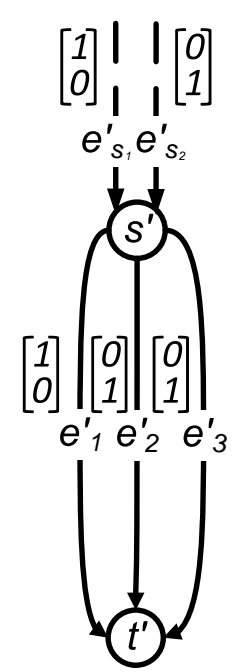

(b)

Fig. 5. Drawbacks of node-based approach

nodes $T$ has $\operatorname{dim}\left(V_{T}\right)=\min (\max f l o w(T), \omega)$. However, this approach, referred to as the nodebased approach, does not accurately capture the independence structure of linear network codes. For example, Figure $5(a)$ is a generic network code and Figure $5(b)$ is a linear dispersion, but the dimensions of $V_{t}$ and $V_{t^{\prime}}$ are the same. Therefore, the node-based approach cannot distinguish between a generic network code and a linear dispersion. However, we notice that these two linear network codes have different regular independent sets. The regular independent sets corresponding to the linear network code in Figure 5(a) are

$$
\left\{e_{1}\right\},\left\{e_{2}\right\},\left\{e_{3}\right\},\left\{e_{1}, e_{2}\right\},\left\{e_{1}, e_{3}\right\},\left\{e_{2}, e_{3}\right\}
$$

while the regular independent sets corresponding to the linear network code in Figure 5(b) are

$$
\left\{e_{1}\right\},\left\{e_{2}\right\},\left\{e_{3}\right\},\left\{e_{1}, e_{2}\right\},\left\{e_{1}, e_{3}\right\} \text {. }
$$

Also, in the node-based representation, different classes of linear network codes cannot be represented in a unified way.

In linear network coding, a fundamental concept is the linear independence among global encoding kernels. We already have obtained necessary and sufficient conditions for a set of global encoding kernels to be possibly linearly independent in Lemma 1 and Lemma 2. Therefore, it 
is possible that different classes of linear network codes can be represented and constructed in a unified way based on these results.

A unified approach for characterizing different classes of linear network codes based on the concept of linearly independence among global encoding kernels is proposed in this section. All the information regarding linearly independence among global encoding kernels is captured by this framework. Specifically, the tool of independence set is used to give the "hologram" of linear network codes in terms of linearly independence. We have already seen in the last section that a generic network code is characterized by regular independent sets. In the rest of this section, we will show that a linear dispersion, a linear broadcast, and a linear multicast can also be characterized by regular independent sets. By using the construction algorithm in Lemma 2 , it is not difficult to see that the construction of different classes of linear network codes can also be unified. The definitions of linear dispersion, linear broadcast and linear multicast are reproduced below for convenience.

Definition 2: [6] A linear network code qualifies as a linear multicast, a linear broadcast, or a linear dispersion respectively, if the following statements hold:

1) $\operatorname{dim}\left(V_{t}\right)=\omega$ for every non-source node $\mathrm{t}$ with $\max f l o w(t) \geq \omega$.

2) $\operatorname{dim}\left(V_{t}\right)=\min (\omega, \max f l o w(t))$ for every non-source node $\mathrm{t}$.

3) $\operatorname{dim}\left(V_{T}\right)=\min (\omega, \max f l o w(T))$ for every collection $\mathrm{T}$ of non-source nodes.

The lemma below establishes the relationship between linear dispersion and regular independent set and gives an alternative definition of linear dispersion in terms of regular independent sets.

Lemma 3 (Linear dispersion): The following two conditions are equivalent for any collection of non-source nodes $T$ in a linear network code.

1) $\operatorname{dim}\left(V_{T}\right)=\min (\max f l o w(T), \omega)$.

2) There exists a size $\min (\max f l o w(T), \omega)$ regular independent set $\xi_{T}$ such that $H e a d(e) \in$ $T$ and $\operatorname{Tail}(e) \notin T$ for any edge $e \in \xi_{T}$.

Proof: 1) $\Rightarrow 2$ ) : Condition 1) means that we can always find a subset $\xi_{T}$ of $\cup_{t \in T} \operatorname{In}(t)$ such that $\left|\xi_{T}\right|=\min (\max f l o w(T), \omega)$ and $\left\{f_{e}: e \in \xi_{T}\right\}$ are linearly independent. Thus, $\xi_{T}$ forms the desired regular independent set.

$2) \Rightarrow 1)$ : Condition 2) implies $\operatorname{dim}\left(V_{T}\right) \geq \min (\max f l o w(T), \omega)$. Using similar argument as in the proof of Lemma 1, we can obtain $\operatorname{dim}\left(V_{T}\right) \leq \min (\max f l o w(T), \omega)$. Thus $\operatorname{dim}\left(V_{T}\right)=$ 
$\min (\max f l o w(T), \omega)$, and 2) implies 1$)$.

In the same manner, we can establish similar results for linear broadcast and linear multicast. The proofs are omitted.

Corollary 1 (Linear broadcast): The following two conditions are equivalent for any nonsource node $t$ in a linear network code.

1) $\operatorname{dim}\left(V_{t}\right)=\min (\max f l o w(t), \omega)$.

2) There exists a size $\min (\max f l o w(t), \omega)$ regular independent set $I_{t}$ such that $H e a d(e)=t$ for any edge $e \in I_{t}$.

Corollary 2 (Linear multicast): The following two conditions are equivalent for any nonsource node $t$ in a linear network code.

1) $\operatorname{dim}\left(V_{t}\right)=\omega$ if $\max f l o w(t) \geq \omega$.

2) There exists a size $\omega$ regular independent set $I_{t}$ such that $\operatorname{Head}(e)=t$ for any edge $e \in I_{t}$ if $\max f l o w(t) \geq \omega$.

When we specialize $\mathcal{I}$ in Lemma 2 to the corresponding independent sets for linear dispersion, linear broadcast, and linear multicast, we can construct a linear dispersion, a linear broadcast, and a linear multicast, respectively. This gives a unified construction algorithm for linear network codes. From Lemma 2 and Corollary 2 , we see that a linear multicast can be constructed provided the field size is no less than $|\mathcal{T}|$ which is the number of receivers. The following example explains these points.

Example 2: The linear network code in Figure 6 is a linear multicast. We observe that the maxflows of nodes 3, 5 and 6 are at least $\omega$ which are equal to two. By Corollary 2, this implies the existence of an associated regular independent set for node 3, 5 and 6 respectively. The associated regular independent set for node 3 is $\left\{f_{e_{3}}, f_{e_{4}}\right\}$; the associated regular independent set for node 5 is $\left\{f_{e_{6}}, f_{e_{9}}\right\}$; the associated regular independent set for node 6 is $\left\{f_{e_{5}}, f_{e_{8}}\right\}$. These three regular independent sets defines a linear multicast.

In general, there can be more than one associated regular independent set for a node $t$ with $\max f l o w(t) \geq \omega$. In that case, any such regular independent set can be chosen to define the linear multicast in terms of regular independent set. 


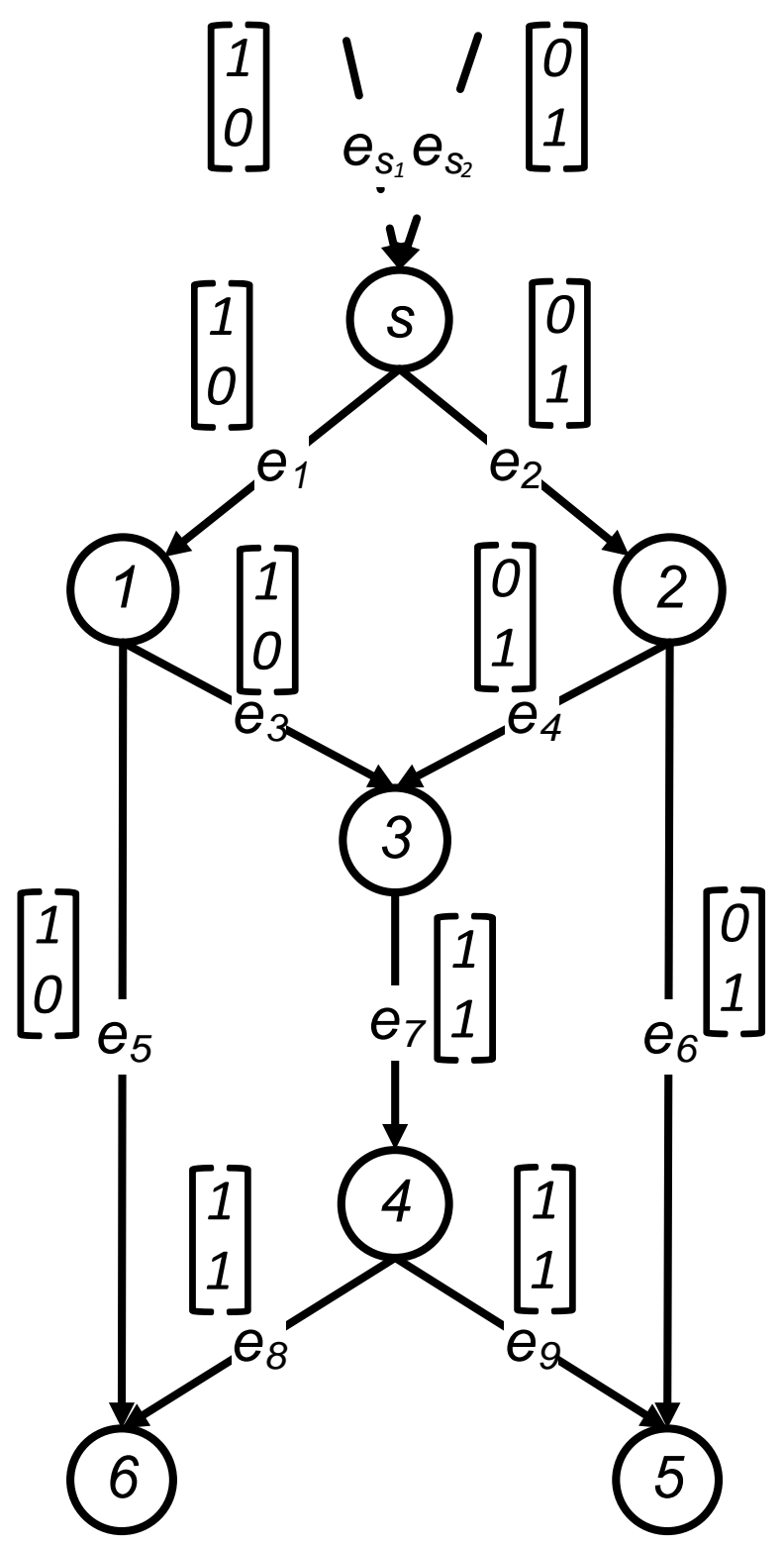

Fig. 6. Linear multicast and regular independent set 


\section{Simplified Proofs}

In this section, we will use the insight obtained in last section to provide simplified proofs for some existing results whose original proofs are complicated. It is not difficult to see that a linear dispersion is a linear broadcast and a linear broadcast is a linear multicast. However, it is not obvious that a generic network code is a linear dispersion. The original proof in [6] for this fact is rather complicated. Here we provide a much simpler proof.

Theorem 2: A generic network code is a linear dispersion.

Proof: A generic network code means that all independent sets are regular. In particular, the corresponding independent sets in Lemma 3 are regular. By the definition of linear dispersion, this linear network code is also a linear dispersion.

For any acyclic graph $G$, by breaking each edge $e_{i}$ into two edges $e_{i}^{1}$ and $e_{i}^{2}$ with $\operatorname{Tail}\left(e_{i}^{1}\right)=$ $\operatorname{Tail}\left(e_{i}\right), \operatorname{Head}\left(e_{i}^{2}\right)=\operatorname{Head}\left(e_{i}\right)$ and $\operatorname{Head}\left(e_{i}^{1}\right)=\operatorname{Tail}\left(e_{i}^{2}\right)=t_{i}^{\prime}$ where $t_{i}$ is a new node inserted in edge $e_{i}$, we obtain an extended graph $G_{E}$. Figure 7 provides one example to illustrate the extended graph $G_{E}$. Now consider any given linear network code defined on the extended graph $G_{E}$. Since node $t_{i}^{\prime}$ has only one incoming edge, we can assume without loss of generality that $f_{e_{i}}^{1}=f_{e_{i}}^{2}$ for all $i$. Then on the original graph $G$, by letting $f_{e_{i}}=f_{e_{i}}^{1}=f_{e_{i}}^{2}$ for all $i$, a linear network code on $G$ is naturally induced by the given linear network code on $G_{E}$. The following Theorem in [7] gives a relationship between generic network code and linear dispersion defined on the original graph and the extended graph, respectively. Again the proof therein is complicated. A simpler proof based on the unified framework is provided here.

Theorem 3: Every linear dispersion on the extended graph $G_{E}$ induces a generic network code on the original graph $G$.

Proof: Let $G$ be the original graph, $G_{E}$ be the extended graph, $\left\{e_{1}, e_{2}, \ldots, e_{m}\right\}$ be any independent set in $G$, and $t_{i}^{\prime}$ be the node inserted in edge $e_{i}$ for $1 \leq i \leq m$. The incoming and outgoing edges of $t_{i}^{\prime}$ are denoted by $e_{i}^{1}$ and $e_{i}^{2}$ respectively. Consider a linear dispersion on the extended graph $G_{E}$ such that $f_{e_{i}}=f_{e_{i}^{1}}=f_{e_{i}^{2}}$ for $1 \leq i \leq m$. This is illustrated in Figure 8. The collection of edges $\left\{e_{1}, e_{2}, \ldots, e_{m}\right\}$ being an independent set on $G$ implies that the collection of edges $\alpha=\left\{e_{1}^{1}, e_{2}^{1}, \ldots, e_{m}^{1}\right\}$ is an independent set on $G_{E}$. Let $T=\left\{t_{1}^{\prime}, t_{2}^{\prime}, \ldots, t_{m}^{\prime}\right\}$. Independent set $\alpha$ is the only independent set with $\operatorname{Head}(e) \in T, \operatorname{Tail}(e) \notin T$ for any $e \in \alpha$ and $|\alpha|=\min (\max f \operatorname{low}(T), \omega)$. Then, by the definition of linear dispersion in Lemma 3, global 


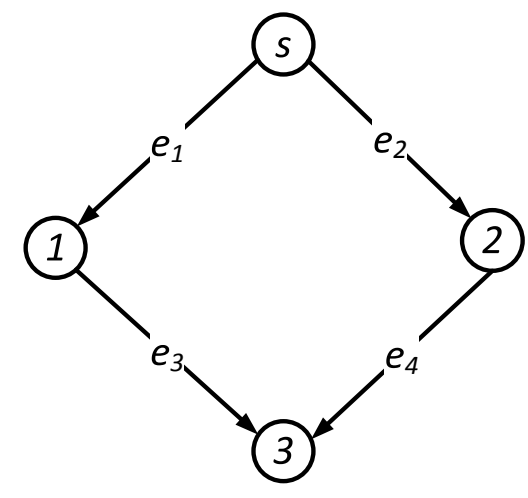

(a) Original graph $G$

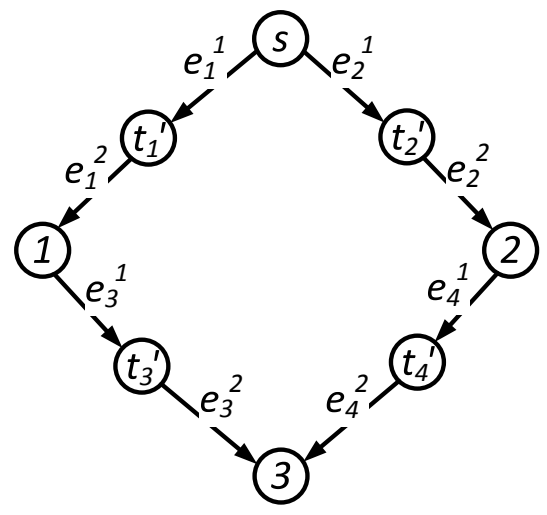

(b) Extended graph $G_{E}$

Fig. 7. Illustration of an extended graph

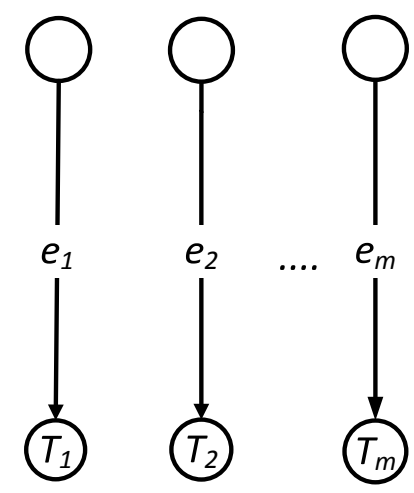

(a) Original graph $G$

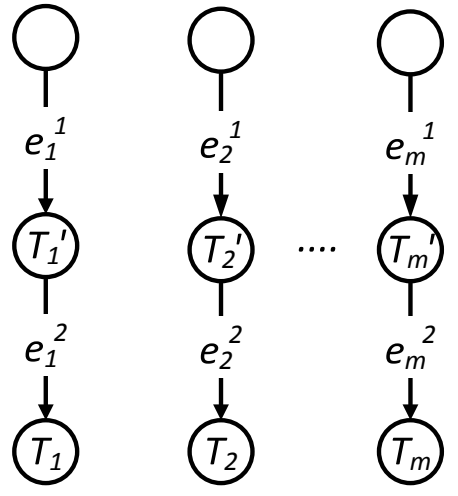

(b) Extended graph $G_{E}$

Fig. 8. A linear dispersion on $G_{E}$ implies a generic network code on $\mathrm{G}$ 
encoding kernels $f_{e_{1}}=f_{e_{1}}^{1}, f_{e_{2}}=f_{e_{2}}^{1}, \ldots, f_{e_{m}}=f_{e_{m}}^{1}$ are linearly independent . This implies that every independent set in $G$ is regular. Hence, we conclude that every linear dispersion on the extended graph $G_{E}$ induces a generic network code on the original graph $G$.

\section{CONCLUSION}

In this paper, the fundamental concept of linear independence among global encoding kernels is studied in depth. Based on this concept, we proved a necessary and sufficient condition for the existence of linear network codes that satisfy certain independence requirement. We proposed and proved the equivalence of several alternative definitions of generic network codes which gives interpretations of generic network codes from different perspectives.

Based on these alternatives definitions of generic network codes, we were able to establish the optimality of generic network codes in terms of linear independence among global encoding kernels. Moreover, we obtained a unified framework for different classes of linear network codes. In particular, this framework suggests a unified construction for such classes of linear network codes.

As applications of our results, we simplified the proofs of some existing results. The results in this paper can potentially be applied to static network codes [11] and network error-correcting codes [12], [13].

\section{ACKNOWLEDGMENT}

The authors would like to thank Prof. Bob Li for his comments.

\section{REFERENCES}

[1] R. Ahlswede, N. Cai, S.-Y. R. Li, and R. W. Yeung, "Network information flow," IEEE Trans. Inf. Theory, vol. 46, no. 4, pp. 1204-1216, Jul. 2000.

[2] S.-Y. R. Li, R. W. Yeung, and N. Cai, "Linear network coding," IEEE Trans. Inf. Theory, vol. 49, no. 2, pp. 371-381, Feb. 2003.

[3] R. Koetter and M. Medard, "An algebraic approach to network coding," IEEE/ACM Trans. Netw., vol. 11, no. 5, pp. 782-795, Oct. 2003.

[4] S. Jaggi, P. Sandrs, P. A. Chou, M. Effros, S. Egner, K. Jain, and L. Tolhuizen, "Polynomial time algorithms for multicast network code construction," IEEE Trans. Inf. Theory, vol. 51, no. 6, pp. 1973 - 1982, Jun. 2005.

[5] T. Ho, B. Leong, M. Medard, R. Koetter, Y. Chang, and M. Effros, "The benefits of coding over routing in a randomized setting," in Proc. IEEE ISIT'03, Jun. 2003. 
[6] R. W. Yeung, S.-Y. R. Li, N. Cai, and Z. Zhang, "Network coding theory," Foundation and Trends in Communications and Information Theory, vol. 2, no. 4 and 5, pp. 241-381, 2005.

[7] P.-W. Kwok and R. W. Yeung, "On the relation between linear dispersion and generic network code," 2006.

[8] R. W. Yeung, Information Theory and Network Coding. Springer, August 2008.

[9] Q.-F. Sun, S.-Y. R. Li, and S.-T. Ho, “On network matroids and linear network codes,” in Proc. IEEE ISIT’08, Jul. 2008.

[10] N. Cai and R. W. Yeung, “Secure network coding,” in Proc. IEEE ISIT'02, Jun. 2002.

[11] R. Koetter and M. Medard, "An algebraic approach to network coding," IEEE/ACM Transactions on Networking, vol. 11, pp. 782-795, 2003.

[12] R. W. Yeung and N. Cai, "Network error correction, part I: basic concepts and upper bounds," Communications in Information and Systems, vol. 6, no. 1, pp. 19 - 36, 2006.

[13] N. Cai and R. W. Yeung, "Network error correction, part II: lower bounds," Communications in Information and Systems, vol. 6, no. 1, pp. $37-54,2006$. 